\title{
Thermoelectric Simulation Using Comsol Multiphysics and Analysis of Contact Resistances Effects
}

\author{
Miguel A. Ramírez, Bs. ${ }^{1}$, Eduardo E. Castillo, PhD. ${ }^{1}$ \\ ${ }^{1}$ Universidad del Turabo, Puerto Rico, ramirez.mramirez.miguel1@gmail.com, ecastillo@suagm.edu
}

\begin{abstract}
The objectives of this work were to develop a simulation of the thermoelectric transport phenomena in a device leg, and to study the effects of the thermal and electrical contact resistances on the temperature difference across the leg. For this simulation Comsol, a finite element software, was used. The results showed that as the thermal resistance between the sample and the electrode increases the temperature difference increases, improving the performance of the device. Changes in electrical contact resistance showed no effect on the temperature difference in the sample; future works will investigate this behavior. The temperature difference was compared with the results of a previous work. Results were found to be in good agreement for low current values, but not for high ones. This difference could be due to the Joule heating effect or to the use of different properties in both works. The results of this work will be extended to model more complex systems, like cooling electronics devices, by using a thermoelectric module or solar-thermoelectric power generation.
\end{abstract}

Keywords_-Thermoelectric, Peltier device, Comsol.

Digital Object Identifier (DOI): http://dx.doi.org/10.18687/LACCEI2015.1.1.170

ISBN: 13 978-0-9822896-8-6

ISSN: $2414-6668$

13 ${ }^{\text {th }}$ LACCEI Annual International Conference: “Engineering Education Facing the Grand Challenges, What Are We Doing?” 


\title{
Thermoelectric Simulation Using Comsol Multiphysics and Analysis of Contact Resistances Effects
}

\author{
Miguel A. Ramírez, Bs, Eduardo E. Castillo, PhD. \\ Universidad del Turabo, Puerto Rico, ramirez.mramirez.miguell@gmail.com, ecastillo@suagm.edu
}

\begin{abstract}
The objectives of this work were to develop a simulation of the thermoelectric transport phenomena in a device leg, and to study the effects of the thermal and electrical contact resistances on the temperature difference across the leg. For this simulation Comsol, a finite element software, was used. The results showed that as the thermal resistance between the sample and the electrode increases the temperature difference increases, improving the performance of the device. Changes in electrical contact resistance showed no effect on the temperature difference in the sample; future works will investigate this behavior. The temperature difference was compared with the results of a previous work. Results were found to be in good agreement for low current values, but not for high ones. This difference could be due to the Joule heating effect or to the use of different properties in both works. The results of this work will be extended to model more complex systems, like cooling electronics devices, by using a thermoelectric module or solarthermoelectric power generation.
\end{abstract}

Keywords-Thermoelectrics, Peltier device, Comsol.

$\begin{array}{ll}\text { NOMENCLATURE } & \\ \mathrm{C}_{\mathrm{p}} & \text { specific heat at constant pressure }(\mathrm{kJ} / \mathrm{kg} \cdot \mathrm{K}) \\ \mathrm{d} & \text { layer thickness }(\mathrm{m}) \\ \mathrm{E} & \text { electric field }(\mathrm{V} / \mathrm{m}) \\ \mathrm{E}_{\mathrm{efm}} & \text { electromotive force }(\mathrm{N} \cdot \mathrm{m} / \mathrm{C}) \\ \mathrm{h} & \text { convective coefficient }\left(\mathrm{W} / \mathrm{m}^{2} \cdot \mathrm{K}\right) \\ \mathrm{h}_{\mathrm{c}} & \text { electrical conductance }(\mathrm{S}) \\ \mathrm{J} & \text { current density }\left(\mathrm{A} / \mathrm{m}^{2}\right) \\ \mathrm{k} & \text { thermal conductivity }(\mathrm{W} / \mathrm{m} \cdot \mathrm{K}) \\ n & \text { normal vector to the interface } \\ \mathrm{Q} & \text { heat sources }(\mathrm{W}) \\ \mathrm{Q}_{\mathrm{i}} & \text { current source }(\mathrm{W}) \\ \mathrm{R} & \text { thermal resistance }\left(\mathrm{m}^{2} \cdot \mathrm{K} / \mathrm{W}\right) \\ \mathrm{S} & \text { Seebeck coefficient }(\mu \mathrm{V} / \mathrm{K}) \\ \mathrm{T} & \text { absolute temperature }(\mathrm{K}) \\ \mathrm{u} & \text { velocity vector }(\mathrm{m} / \mathrm{s}) \\ \mathrm{V} & \text { electric potential }(\mathrm{V})\end{array}$

Greek Symbols

$\begin{array}{ll}\nabla & \text { Del operator } \\ \Pi_{\mathrm{A}} & \text { Peltier coefficient of conductor A (W/A) } \\ \Pi_{\mathrm{B}} & \text { Peltier coefficient of conductor B (W/A) } \\ \rho & \text { Electrical resistivity }(\Omega \cdot \mathrm{m}) \\ \sigma & \text { electric conductivity }(\mathrm{S} / \mathrm{m})\end{array}$

Subscripts

e external

$\begin{array}{ll}\mathrm{d} & \text { downside pair } \\ \mathrm{u} & \text { upside pair } \\ \mathrm{s} & \text { surface } \\ \mathrm{ext} & \text { external } \\ 1,2 & \text { sides of boundary condition }\end{array}$

\section{INTRODUCTION}

In the recent years the development of the thermoelectric materials has been of great interest due to their multiples applications. A thermoelectric (Fig. 1) is a device that converts a temperature difference into voltage or vice versa. Thermoelectrics can be used whenever there is heat waste (like hot pipes, engines, satellites chips, etc.) to produce energy that can be used to power other devices. Other applications of the thermoelectrics are in cooling systems for water, wine, computers chips and lasers diodes[1].

Thermoelectric systems have many advantages. They are simple systems that work in harsh environments, quiet in operation, capable of virtually endless shelf life. They have a simple structure without any moving parts, extremely reliable and can be driven by low grade heat energy [2]. Despite its low energy conversion efficiency, thermoelectric generators are under development and study. Different types of solar energy concentrators combined with thermoelectric generators have been tested [2].

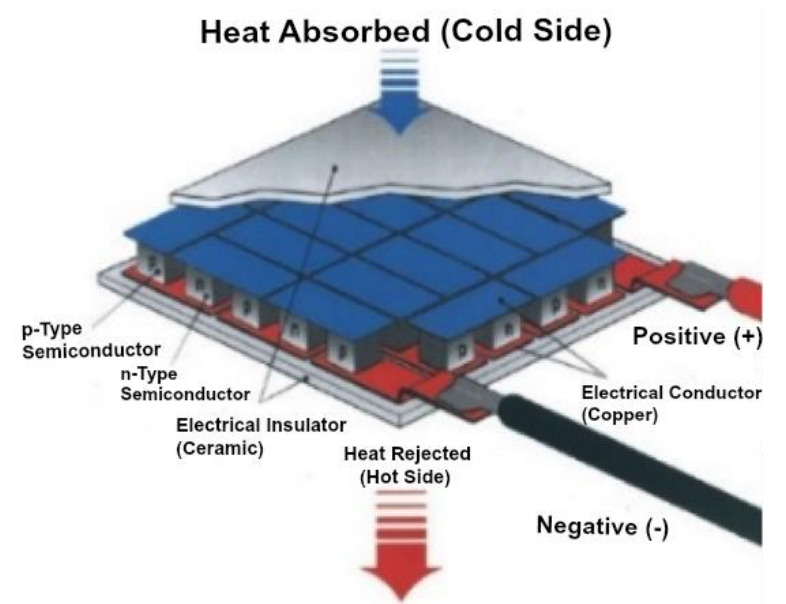

Fig. 1 Thermoelectric device model

S. Maneewan developed a thermoelectric roof solar collector to generate the power to move a fan. He was looking

$1^{\text {th }}$ LACCEI Annual International Conference: “Engineering Education Facing the Grand Challenges, What Are We Doing?” July 29-31, 2015, Santo Domingo, Dominican Republic ISBN: 13 978-0-9822896-8-6

ISSN: $2414-6668$ 
for a simple alternative for enhancing attic/room ventilation which can help reducing heat accumulation in the house [3]. R. Amatya determined in his study that solar thermoelectrics can be economically competitive with small-scale PV power generation for many applications such as power supply for remote sensors [4]. In his study, he was using a thermoelectric module design with longer TE legs, low parasitic, and materials with improved thermoelectric properties at high temperature.

Materials with different thermoelectric properties are under study in order to improve their applications. For example, the incorporation of skutterudite to solar thermoelectric generators material for near sun missions was proposed because thermoelectrics are less sensitive to temperature and radiation damages than the traditionally used solar cells [5]. Other studies determined that pressure application leads to a significant improvement in the thermoelectric efficiency of Bismuth Telluride alloy, $\mathrm{Bi}_{2} \mathrm{Te}_{3}$ [6].

Thermoelectrics used for power generation are based on the Seebeck effect and the ones employed for cooling function are based on the Peltier effect [7]. In both cases, the Joule heating is taken in to account.

The Seebeck effect is the conversion of a temperature difference between two conductor materials to voltage [7]. As seen in Fig. 2, when an n-type and p-type thermoelectric materials are in contact and their edges are exposed to a temperature difference, they generate a voltage. The Seebeck effect is described by an electromotive force as follows:

$E_{\text {efm }}=-S \nabla T$

The Seebeck effect varies mainly by the temperature difference and depends on the material of the conductor used. A good example of this effect is the measurement of temperature by a thermocouple where the voltage is measured and correlated with the temperature.

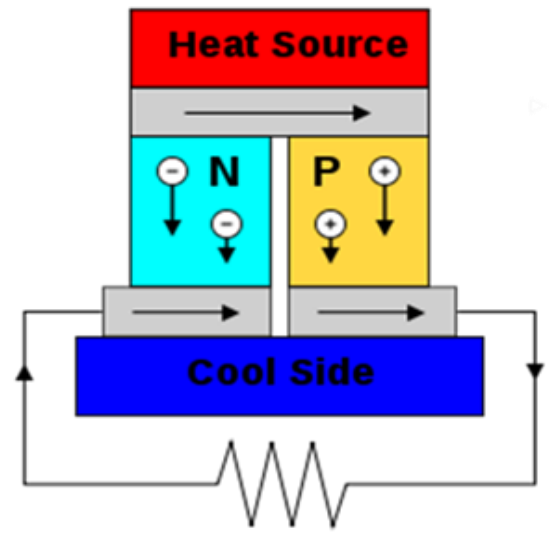

Fig. 2 Seebeck effect

The Peltier effect is the presence of heating or cooling at the electrified junction of two different conductors [7]. The Peltier effect states that when electric current flows through two dissimilar conductors, heat is removed or added at the junction points (see Fig. 3). For two conductors, A and B, this effect is described by:

$\mathrm{Q}=\left(\Pi_{\mathrm{A}}-\Pi_{\mathrm{B}}\right) \mathrm{I}$.

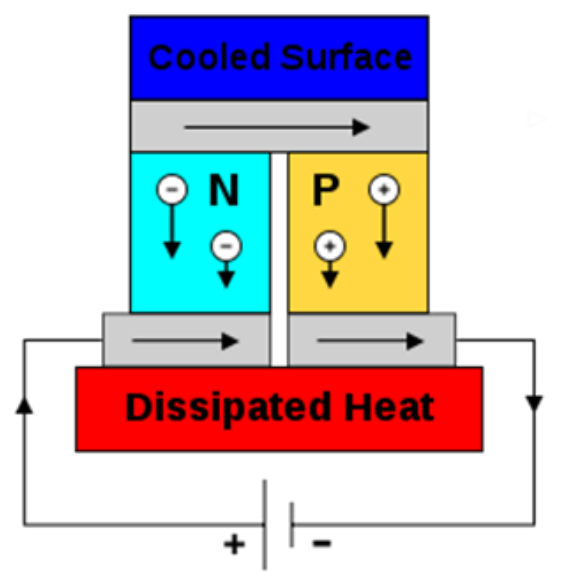

Fig. 3 Peltier effect

The Peltier coefficient represents how much heat is carried per unit charge. It is important to mention that the heat generated at the junction is influenced by the Joule heating and thermal gradient effects. The Peltier effect can be considered the counterpart of the Seebeck effect. A closed thermoelectric circuit creates a current by Seebeck effect, while the Peltier effect drives heat from one hot surface to a cool surface.

The Joule heating effect is the process by which the flow of current through a conductor produces heat. It is described by:

$\mathrm{Q}=\mathrm{I}^{2} \mathrm{R}$.

The main goals of this research were to investigate the effects of: 1) Seebeck, Peltier and Joule heating effects; 2) electrical and thermal contact resistances; and 3) current, in to the performance of the thermoelectric device by using COMSOL multyphysics software.

\section{Mathematical Model}

\section{A. Governing equations}

The current conservation equation was applied using the software to calculate the electric field and the current density as follows:

$\mathrm{E}=-\nabla \mathrm{V}$

$\mathrm{J}=\sigma \mathrm{E}+\mathrm{J}_{\mathrm{e}}$ 
$\nabla \mathrm{J}=\mathrm{Q}_{\mathrm{j}}$

The boundary conditions for these equations are:

$n \mathrm{~J}_{1}=\mathrm{h}_{\mathrm{c}}\left(\mathrm{V}_{1}-\mathrm{V}_{2}\right)$

$n \mathrm{~J}_{2}=\mathrm{h}_{\mathrm{c}}\left(\mathrm{V}_{2}-\mathrm{V}_{1}\right)$.

The equation of energy conservation for heat transfer rate is:

$\rho \mathrm{C}_{\mathrm{p}} \mathrm{u} \times \nabla \mathrm{T}=\nabla \times(\mathrm{k} \nabla \mathrm{T})+\mathrm{Q}$.

The thermal resistance boundary conditions are:

$-n_{d} \times\left(-k_{d} \nabla T_{d}\right)=-\left(T_{u}-T_{d}\right) / R_{s}$

$-n_{u} \times\left(-k_{u} \nabla T_{u}\right)=-\left(T_{d}-T_{u}\right) / R_{s}$

$R_{s}=d_{s} / K_{S}$

The convective heat transfer boundary condition equation is:

$-\mathrm{n} \times(-\mathrm{k} \nabla \mathrm{T})=\mathrm{h} \times\left(\mathrm{T}_{\mathrm{ext}}-\mathrm{T}\right)$.

\section{B. Dimensions and materials}

The components of the thermoelectric device are: (1) electrode, (2) spreader and (3) sample. The electrode is used to apply the current across the sample. The spreader makes the current uniform when it enters the sample and makes the heat flux unidimensional. The sample is the thermoelectric material under evaluation, in this case Bismuth Telluride alloy. A diagram of the different components of the thermoelectric device is shown on Fig. 4.

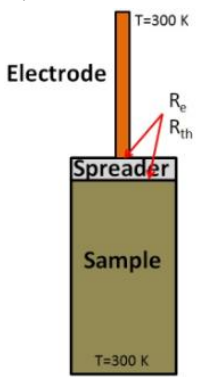

Fig.4 Components of thermoelectric device: (1) electrode, (2) spreader, (3) sample.

The dimensions of the different components of the thermoelectric device are shown in Table 1. Materials and materials properties are defined in Table 2 .

\section{Assumptions and additional boundary conditions}

Steady state conditions were assumed. Also, it was assumed that the substrate under the sample was at room temperature due to its high heat capacity as well as the big mass of the connector at the end of the electrode. So, a temperature of $300 \mathrm{~K}$ was set at the bottom surface of the sample as well as at the top end of the electrode. The bottom surface of the sample was used as electrical ground while the top edge of the electrode was used to introduce the current. Thermal contact resistance of $1 \times 10^{-6} \mathrm{~m}^{2} \cdot \mathrm{K} / \mathrm{W}$ and electrical contact resistance of $1 \times 10^{-12} \mathrm{~m}^{2} \cdot \Omega$, were used on both, electrode-spreader and spreader-sample, interfaces. These are typical values found in the literature of thermoelectric modules [8]. Natural convection was established for the outside boundaries of the system with air temperature of 300 $\mathrm{K}$ and a convective heat transfer coefficient of $5 \mathrm{~W} / \mathrm{m}^{2} \cdot \mathrm{K}$.

Table 1 Dimensions of the components

\begin{tabular}{|l|c|c|c|c|}
\hline Geometry & Width & Height & Depth & Diameter \\
\hline Electrode (1) & & 10 & & 0.075 \\
\hline Spreader (2) & 1 & 0.05 & 1 & \\
\hline Sample (3) & 1 & 2 & 1 & \\
\hline
\end{tabular}

* All the dimensions are in $\mathrm{mm}$.

Table 2 Materials and properties of the components

\begin{tabular}{|l|l|c|c|c|}
\hline \multicolumn{1}{|c|}{ Geometry } & Material & $\begin{array}{c}\mathrm{k} \\
\mathrm{W} / \mathrm{m} \cdot \mathrm{K}\end{array}$ & $\begin{array}{c}\mathrm{S} \\
\mu \mathrm{V} / \mathrm{K}\end{array}$ & $\begin{array}{c}\underline{\rho} \\
\mu \cdot \mathrm{m}\end{array}$ \\
\hline Electrode (1) & Copper & 400 & 6.5 & 0.016 \\
\hline Spreader (2) & Indium & 80 & 1 & 0.830 \\
\hline Sample (3) & $\mathrm{Bi}_{2} \mathrm{Te}_{3}$ & 1.5 & -210 & 10.0 \\
\hline
\end{tabular}

\section{RESULTS AND DISCUSSION}

The study of the thermoelectric device was divided into several parts. Initially, the temperature profile of the system was examined under the two different directions of the current. Then, the effect of the electrical and thermal contact resistances on the temperature at different vertical points through the sample was examined. Finally, the results for this simulations were compared with the experimental results of a previous work [8].

Graphs of the temperature profile in the system, and temperature difference between the electrode-spreader interface and the bottom surface of the sample were investigated for several conditions.

Fig. 5 shows the temperature distribution along the center line of the system, starting at the bottom of the sample. The thermal and electrical contact resistances used were $6.04 \times 10^{-}$ ${ }^{6} \mathrm{~m}^{2} \cdot \mathrm{K} / \mathrm{W}$ and $1.66 \times 10^{-12} \mathrm{~m}^{2} \cdot \Omega$, respectively. A current of $-0.5 \mathrm{~A}$ was applied (the negative sign indicates that the current enters at the bottom of the sample directed towards the electrode). Also, Fig. 5 shows the relative location of the

$13^{\text {th }}$ LACCEI Annual International Conference: "Engineering Education Facing the Grand Challenges, What Are We Doing?" July 29-31, 2015, Santo Domingo, Dominican Republic 
three components of the device. The bottom surface of the sample has a higher temperature than its top surface, as evidenced by the negative slope of the first part of the curve. This cooling effect is due to the dominance of the Peltier effect in the sample, the current direction and the Seebeck coefficient of the sample. The second area of the curve, where the temperature starts to increase, represents the change in temperature across the spreader. The higher and positive slope area corresponds to the changes in temperature at the electrode. The temperature in the second and third areas increases until it reaches the boundary condition of $300 \mathrm{~K}$ at the top of the electrode. The leading effect in these areas is the heat conduction.

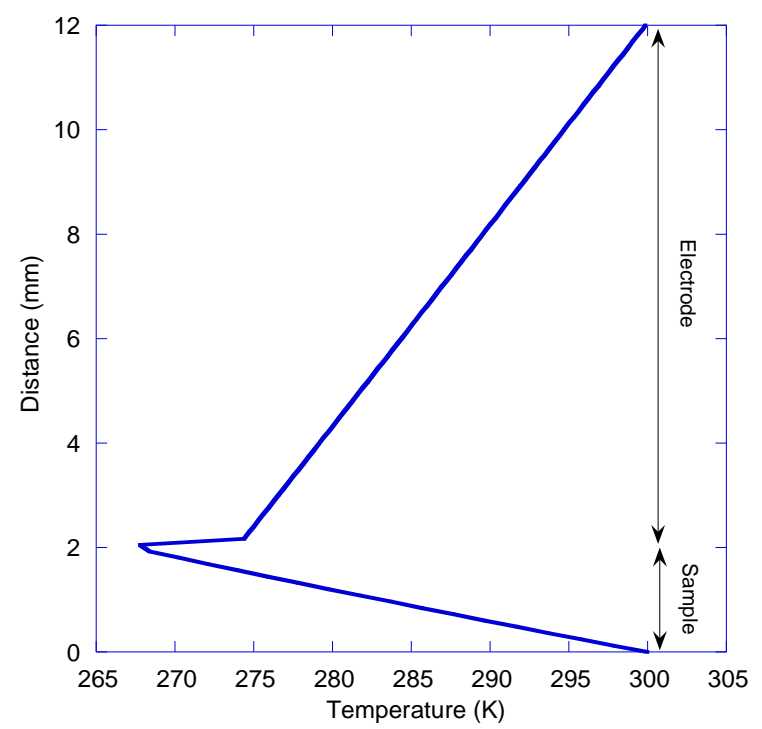

Fig. 5. Temperature profile along the center line of the system. A current of $0.5 \mathrm{~A}$ was applied at the bottom of the sample. Thermal and electrical contact resistances used were $6.04 \times 10^{-6} \mathrm{~m}^{2} \cdot \mathrm{K} / \mathrm{W}$ and $1.66 \times 10^{-12} \mathrm{~m}^{2} \cdot \Omega$, respectively.

Fig. 6 shows a closed up of the Fig. 5 at the spreader area. Due to the Peltier effect, the temperature in the sample decreases linearly from the bottom to the sample-spreader interface, located at $2.0 \mathrm{~mm}$. Then, there is a small jump due to the interface thermal resistance and a slight parabolic profile in the spreader due to the Joule heating. The temperature jumps (slightly horizontal portion of the graph) to higher values at the spreader-electrode interface, at $2.05 \mathrm{~mm}$, due the interface thermal resistance.

Fig. 7 shows the temperature distribution along the center line of the system, starting at the bottom of the sample. Thermal and electrical contact resistances values are as previously; but a current of $0.5 \mathrm{~A}$ was applied at the electrode directed towards the bottom of the sample. In this case, as stated by the Peltier effect, the top of the sample was heated. In the heated region, the behavior of the temperature profile was similar to the previous case.

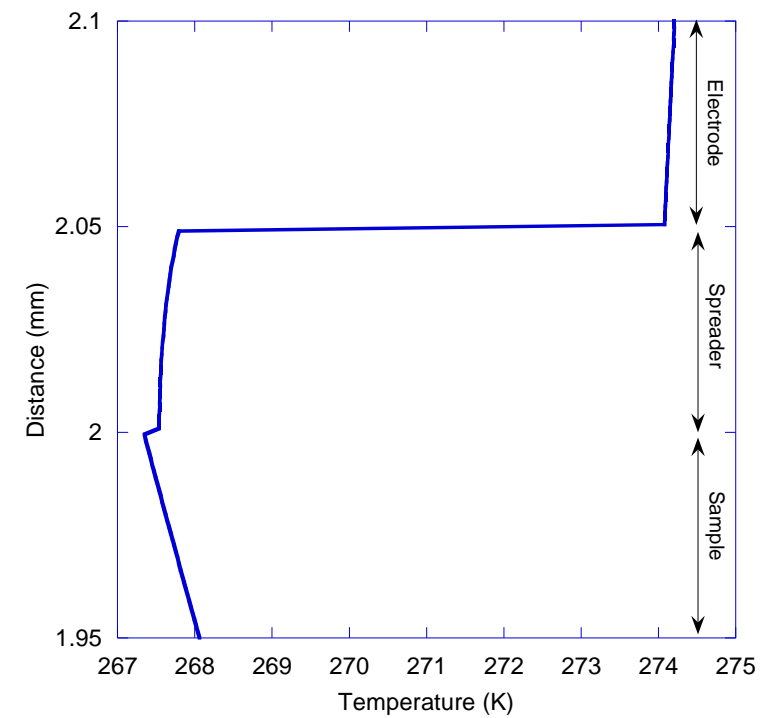

Fig. 6. Temperature variation along the center line of the system, closed up of Fig. 5 at the spreader area.

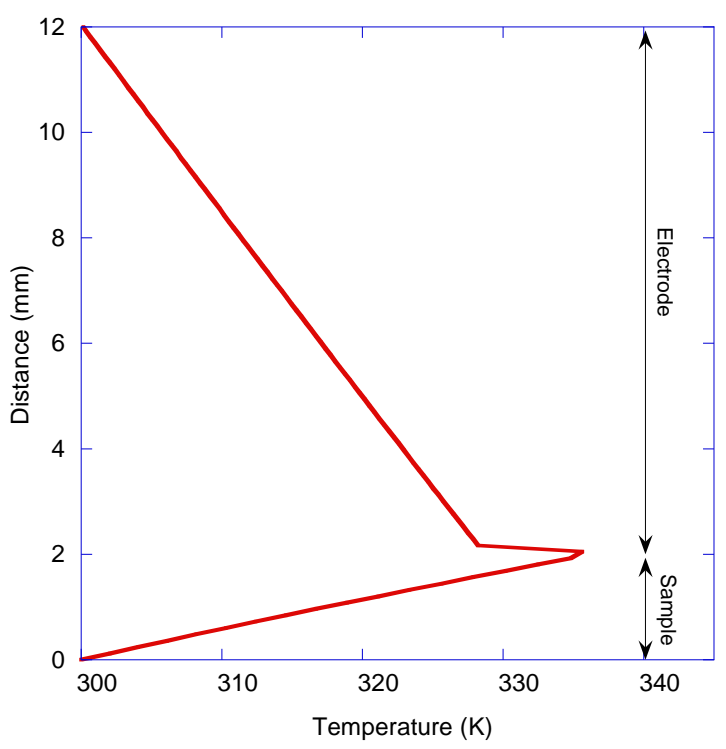

Fig. 7. Temperature profile along the center line of the system. A current of $0.5 \mathrm{~A}$ was applied at the top of the electrode. Thermal and electrical contact resistances used were $6.04 \times 10^{-6} \mathrm{~m}^{2} \cdot \mathrm{K} / \mathrm{W}$ and $1.66 \times 10^{-12} \mathrm{~m}^{2} \cdot \Omega$, respectively.

Fig. 8 shows a closed up of the Fig. 7 at the spreader area. Due to the Peltier effect, the temperature at the sample increases linearly from the bottom toward the sample-spreader interface located at $2.0 \mathrm{~mm}$. Then, there is a small jump due to the interface thermal resistance and a slight parabolic profile in the spreader due to the Joule heating. The temperature jumps to lower values at the spreader-electrode interface, $2.05 \mathrm{~mm}$, due the interface thermal resistance. 


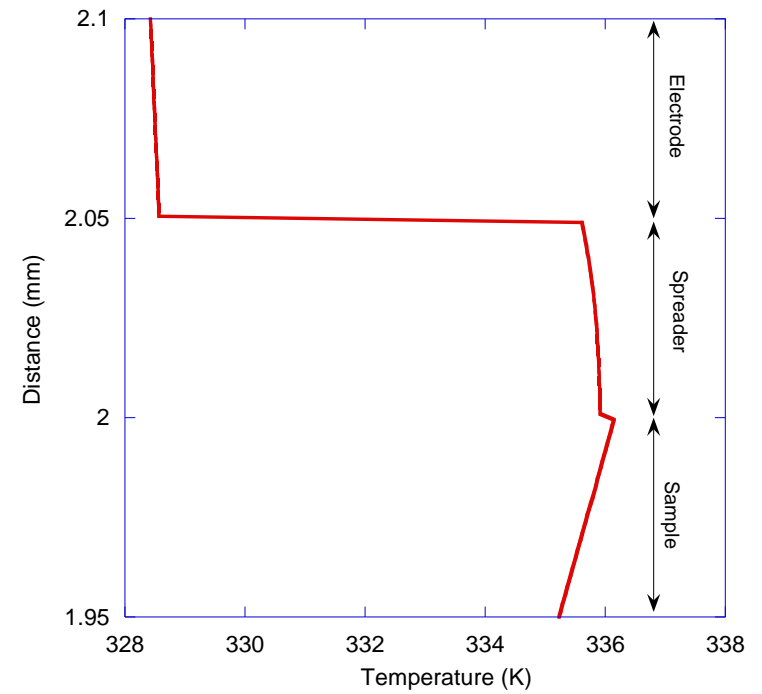

Fig. 8. Temperature variation along the center line of the system, closed up of Fig. 7 at the spreader area.

Fig. 9 shows the temperature difference between the top surface of the spreader and the bottom surface of the sample, with respect to the current. This analysis used a current range from $-0.5 \mathrm{~A}$ to $0.5 \mathrm{~A}$; thermal and electrical contact resistances were $6.04 \times 10^{-6} \mathrm{~m}^{2} \cdot \mathrm{K} / \mathrm{W}$ and 0 , respectively. These values were chosen from the previous research [8]. Fig. 9 shows that when the current was applied at the bottom of the sample (negative values) the top surface was cooled (the bottom surface temperature was fixed at $300 \mathrm{~K}$ ); however, this top surface was heated when the current goes in the opposite direction. As seen on Fig. 9, for $-0.5 \mathrm{~A}$ the cooling effect across the sample was $32 \mathrm{~K}$ and for $0.5 \mathrm{~A}$ the heating was 36 $\mathrm{K}$. The heating effect was bigger than the cooler effect due to the Joule heating.

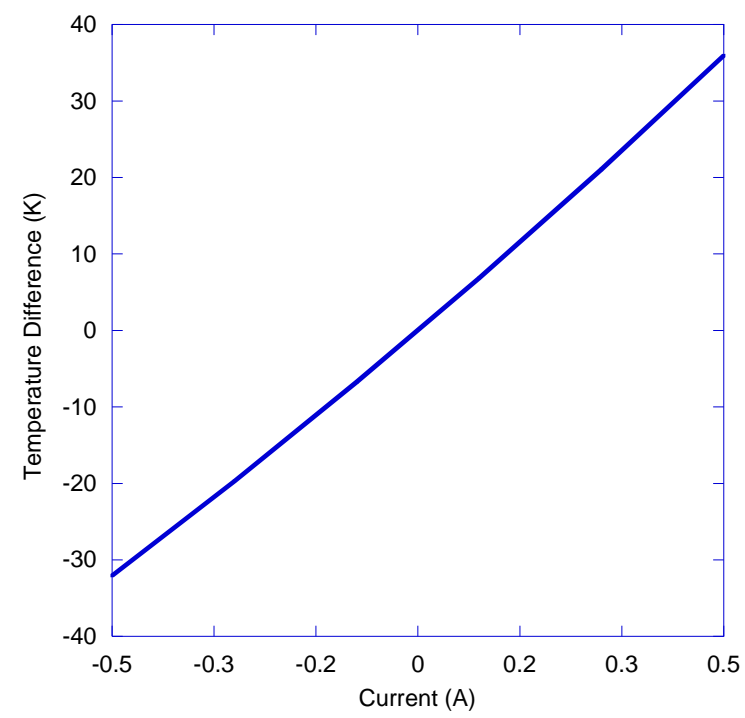

Fig.9. Temperature difference across the sample respect to current. The thermal and electrical contact resistances were $6.04 \times 10^{-6} \mathrm{~m}^{2} \cdot \mathrm{K} / \mathrm{W}$ and 0 , respectively.
Fig. 10 shows the temperature difference between the top surface of the spreader and the bottom surface of the sample with respect to the current. This time, the thermal and electrical resistances were 0 and $1.66 \times 10^{-12} \mathrm{~m}^{2} \cdot \Omega$, respectively. Fig. 10 shows that as the current increases the temperature gradient increases almost linearly.

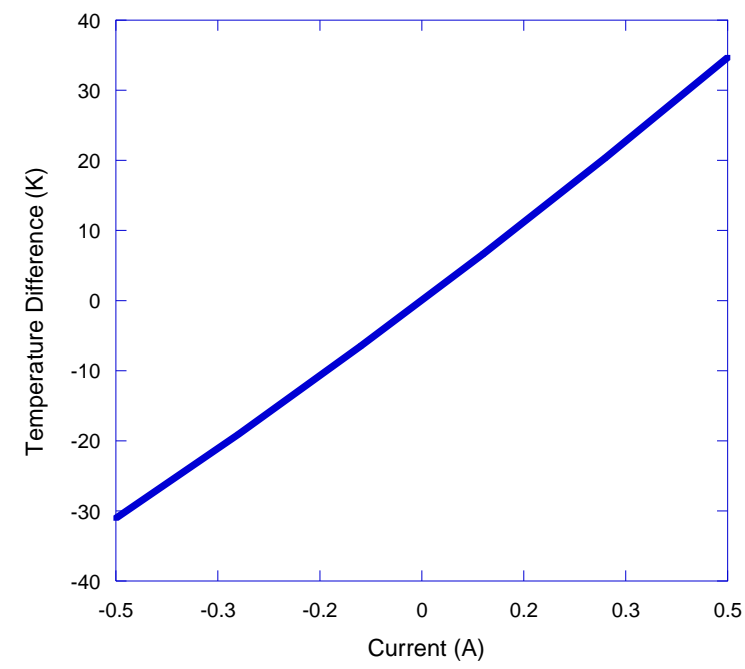

Fig.10. Thermal and electrical resistance of 0 and $1.66 \times 10^{-12} \mathrm{~m}^{2} \Omega$, respectively.

Fig. 11 shows the temperature difference in the sample with respect to current for different values of thermal resistance while the electrical resistance was 0 . These curves show that as the thermal resistance increases the temperature gradient increases.

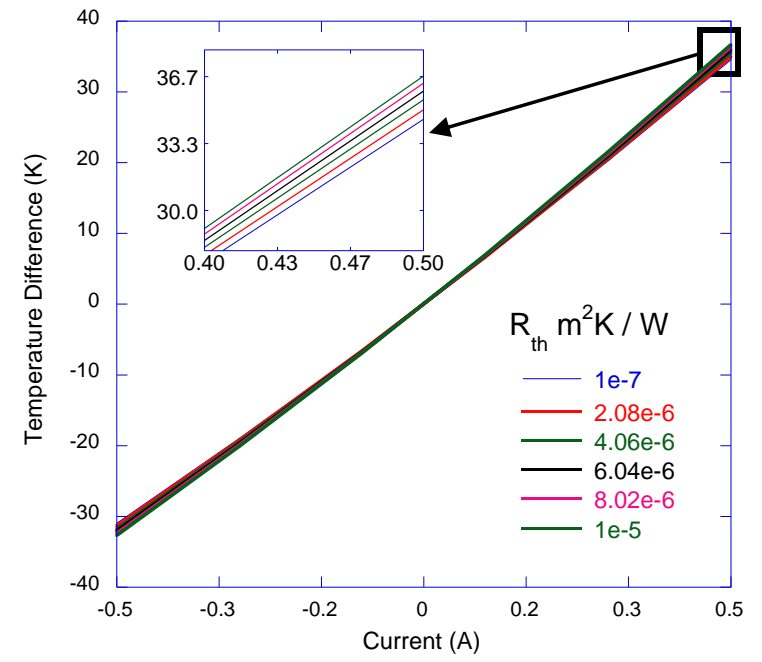

Fig.11. Effects of the thermal contact resistance on the temperature difference across the sample. Electrical contact resistance was zero.

Fig. 12 shows the temperature difference in the sample with respect to current, for different values of electrical contact resistance. The thermal resistance used was zero. The graph shows that the electrical resistance does not have any influence on the temperature difference; which is contradictory because as the electrical resistance increases the 
Joule heating increases, decreasing the temperature difference. This result could be due to the small area of the electrode.

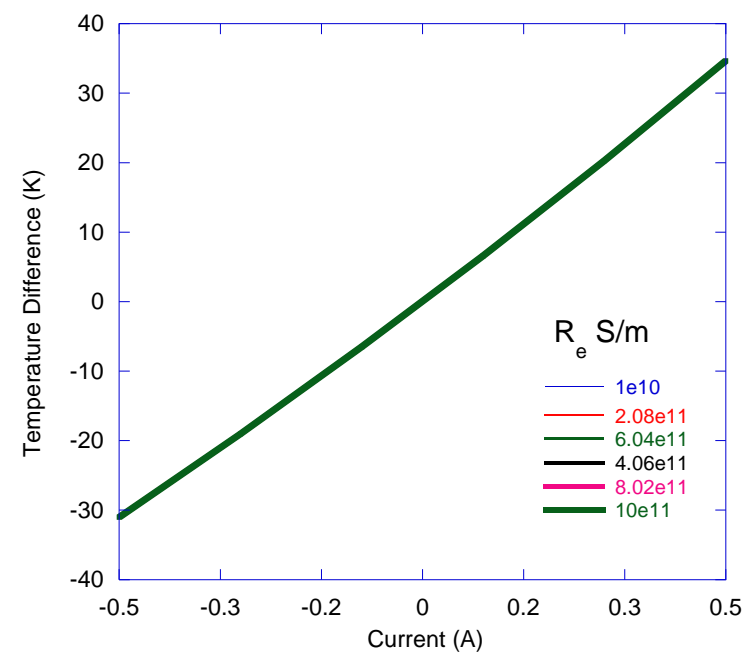

Fig.12. Effects of the electrical contact resistance on the temperature difference across the sample. Thermal contact resistance was zero.

Fig. 13 shows the temperature of the center point of the top surface of the spreader as a function of the thermal contact resistance, for several values of current. The electrical contact resistance was zero. As before, the graph shows that as the magnitude of the current increases, the temperature difference in the sample increases (the bottom of the sample is at $300 \mathrm{~K}$ ).

Moreover, as shown in Fig. 13, there is a small change in temperature for small values of the thermal contact resistance. When the resistance is approximately $2 \times 10^{-6} \mathrm{~m}^{2} \cdot \mathrm{K} / \mathrm{W}$ the slope of the graphs change. This behavior is explained by the fact that the thermal contact resistance is approximately equal to the thermal resistance of the sample.

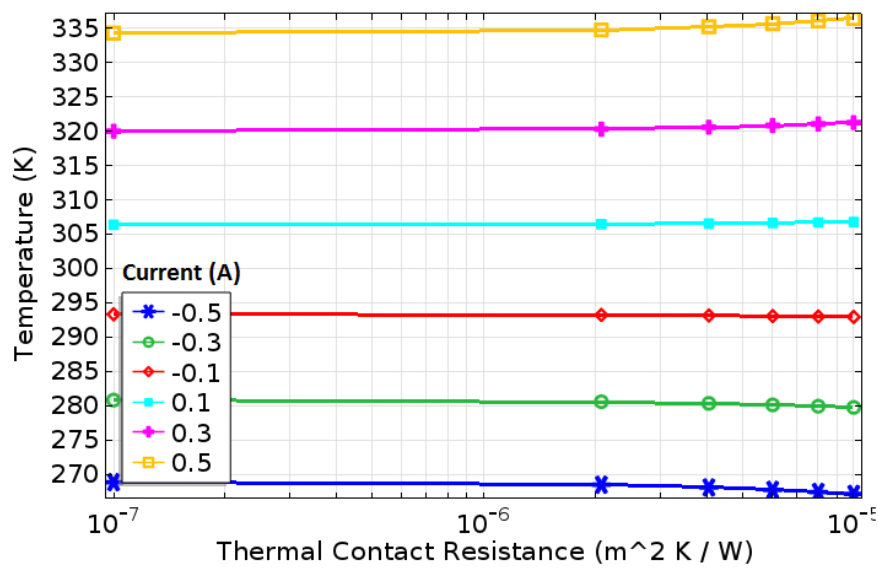

Fig.13. Effects of the thermal contact resistance and current on the temperature of the center point of the top surface of the spreader. Electrical contact resistance was zero.

Fig. 14 shows the temperature of the center point of the top surface of the spreader as a function of the electrical contact conductance, for several values of current. The thermal contact resistance was zero. As before, the graph does not show any influence of the electrical contact conductance on the temperature at the top surface of the spreader.

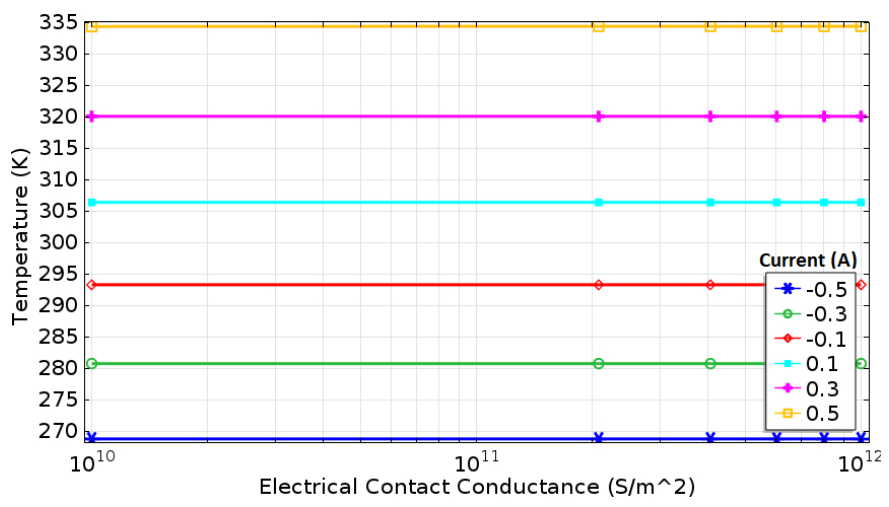

Fig.14. Effects of the electrical contact conductance and current on the temperature of the center point of the top surface of the spreader. Thermal contact resistance was zero.

Finally, the temperatures obtained in this study were compared with the experimental values of a previous research [8]. Fig.15 compares the Comsol and the experimental temperature differences across the sample, in a range of currents according to the experimental results. The Comsol and experimental results were very similar for small magnitude of the current. The difference between the results increases for higher current values. This discrepancy could be due to the effects of the Joule heating or some inconsistencies in the properties used for both studies.

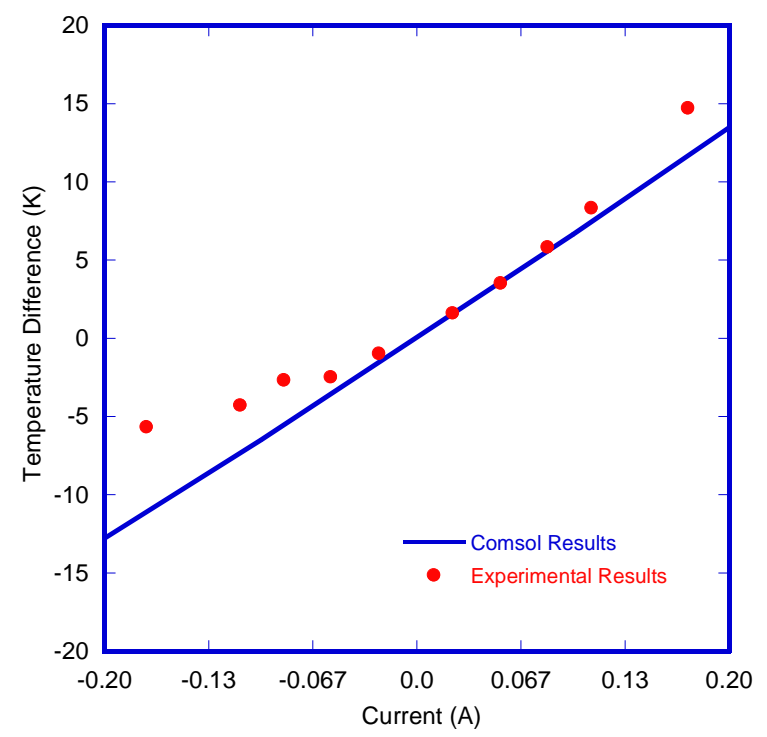

Fig.15. Temperature difference across the sample for this study (Comsol) and experimental results of a previous work[8].

$13^{\text {th }}$ LACCEI Annual International Conference: “Engineering Education Facing the Grand Challenges, What Are We Doing?" 


\section{CONCLUSIONS}

The transport thermoelectric phenomenon was simulated by using COMSOL (a finite element software); taking account the effects of the Peltier, Seebeck and Joule heating effects. The electrical and thermal contact resistances were also simulated by using models of thin layers in the software. The results showed that as the thermal resistance between the sample and the electrode increases the temperature difference increases, improving the performance of the device. The temperature difference was not affected by the electrical contact resistance and this behavior will be explored in a future research.

The results of this work were very similar to the experimental results of a previous work for low current values; but not for high values. For high current, the Joule heating effect in the sample and its contacts plays an important role, compared with the Seebeck effect and heat conduction. So, small differences in the properties or boundary conditions between both works could generate differences in the temperature profile. The analysis performed in this research will be used as a base to explore more complex systems, like cooling electronic devices, by using a thermoelectric module or solar-thermoelectric power generation.

\section{REFERENCES}

[1] H. Xi, L. Luo, and G. Fraisse, "Development and applications of solarbased thermoelectric technologies," Renew. Sustain. Energy Rev., vol. 11, no. 5, pp. 923-936, Jun. 2007.

[2] T. M. Maslamani and M. a Majid, "Development of Solar Thermoelectric Generator," vol. 10, no. 9, pp. 123-134, 2014.

[3] S. Maneewan, J. Khedari, B. Zeghmati, J. Hirunlabh, and J. Eakburanawat, "Investigation on generated power of thermoelectric roof solar collector," Renew. Energy, vol. 29, no. 5, pp. 743-752, Apr. 2004.

[4] R. Amatya and R. J. Ram, "Solar thermoelectric generator for micropower applications," J. Electron. Mater., vol. 39, no. 9, pp. 17351740,2010

[5] H. Scherrer, L. Vikhor, B. Lenoir, a. Dauscher, and P. Poinas, "Solar thermolectric generator based on skutterudites," J. Power Sources, vol. 115, no. 0378, pp. 141-148, 2003.

[6] S. V. Ovsyannikov, V. V. Shchennikov, G. V. Vorontsov, A. Y. Manakov, A. Y. Likhacheva, and V. a. Kulbachinskii, "Giant improvement of thermoelectric power factor of Bi2 Te3 under pressure," J. Appl. Phys., vol. 104, no. 5, p. 053713, 2008.

[7] L. E. Bell, "Cooling, heating, generating power, and recovering waste heat with thermoelectric systems," Sci., vol. 321, no. 5895, pp. 14571461, Sep. 2008.

[8] E. E. Castillo, C. L. Hapenciuc, and T. Borca-Tasciuc, "Thermoelectric characterization by transient Harman method under nonideal contact and boundary conditions," Rev. Sci. Instruments, vol. 81, no. 4, p. 044902, Apr. 2010.

$13^{\text {th }}$ LACCEI Annual International Conference: "Engineering Education Facing the Grand Challenges, What Are We Doing?" July 29-31, 2015, Santo Domingo, Dominican Republic 